\title{
Targeted genome-wide investigation identifies novel SNPs associated with diabetic nephropathy
}

\author{
Amy Jayne McKnight • Diane Currie • Chris C. Patterson • \\ Alexander P. Maxwell - Damian G. Fogarty • \\ The Warren 3/UK GoKinD Study Group
}

Received: 14 July 2009/Revised: 23 October 2009/Accepted: 8 February 2010/Published online: 24 February 2010

(C) Springer Science+Business Media B.V. 2010

\begin{abstract}
Loci contributing to complex disease have been identified by focusing on genome-wide scans utilising nonsynonymous single nucleotide polymorphisms (nsSNPs). We employed Illumina's HNS12 BeadChip (13,917 highvalue SNPs) which was specifically designed to capture nsSNPs and ideally complements more dense genome-wide association studies that fail to consider many of these putatively functional variants. The HNS12 panel also includes 870 tag SNPs covering the major histocompatibility region. All individuals genotyped in this study were Caucasians with (cases) and without (controls) diabetic nephropathy. About 449 individuals with type 2 diabetes (203 cases, 246 controls) were genotyped in the initial study. 1,467 individuals with type 1 diabetes (718 cases, 749 controls) were genotyped in the follow up study. 11,152 SNPs were successfully analysed and ranked for association with diabetic nephropathy based on significance $(P)$ values. The top ranked 32 SNPs were subsequently genotyped using MassARRAY iPLEX ${ }^{\mathrm{TM}}$ and TaqMan technologies to investigate association of these polymorphisms with nephropathy in individuals with type
\end{abstract}

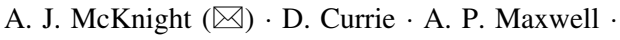

D. G. Fogarty

Nephrology Research Groups, Queen's University of Belfast, c/o Regional Genetics Centre, Level A, Tower Block, Belfast City Hospital, Lisburn Road, Belfast BT9 7AB,

Northern Ireland, UK

e-mail: a.j.mcknight@qub.ac.uk

C. C. Patterson

Epidemiology Research Groups, Centre for Public Health,

Queen's University of Belfast, Belfast, UK
}

1 diabetes. The top ranked nsSNP, $\operatorname{rs} 1543547\left(P=10^{-5}\right)$, is located in RAETIL, a major histocompatibility class Irelated gene at $6 \mathrm{q} 25.1$. Of particular interest, multiple nsSNPs within the top ranked $(0.2 \%)$ SNPs are within several plausible candidate genes for nephropathy on $3 \mathrm{q} 21.3$ and $6 \mathrm{p} 21.3$.

Keywords nsSNP $\cdot$ MHC $\cdot$ Genetic predisposition · Diabetic nephropathy

\section{Introduction}

Diabetic nephropathy is the leading cause of end stage renal disease (ESRD), accounting for $22 \%$ of incident patients with ESRD in the UK (Ansell et al. 2008) and 44\% in USA (USRDS 2008). There is a genetic predisposition to diabetic nephropathy (Savage et al. 2007), although refined genetic loci in White individuals have not yet been convincingly confirmed across multiple populations (McKnight et al. 2009a).

Non-synonymous single nucleotide polymorphisms (nsSNPs) are distributed throughout the human genome and are functionally relevant as they generate changes in amino acid sequences for proteins. These nsSNPs are important contributors to Mendelian and complex disease phenotypes (Stitziel et al. 2004). Several studies have successfully identified loci contributing to complex disease by focusing on a genome-wide panel of nsSNPs (Smyth et al. 2006; Hampe et al. 2007; WTCCC 2007). Although our recent nsSNP study for diabetic nephropathy did not identify variants that contribute significantly to the development of diabetic nephropathy, this research was limited in scope to only 1,111 nsSNPs in 1,021 genes (Savage et al. 2008). 
We report association data with diabetic nephropathy for the HNS12 BeadChip (13,917 SNPs, Illumina Inc, San Diego, CA, USA). This HNS12 panel resulted from an in-depth focus on high-value, gene-centric variants and ideally complements more dense genome-wide association studies that fail to consider many of these variants (Evans et al. 2008). This panel comprises more than 11,600 nsSNPs across the genome, and a dense set of 870 tag SNPs across the major histocompatibility region (MHC).

\section{Results}

Clinical characteristics of recruited individuals are summarised in Table 1. As expected with diabetic nephropathy, higher blood pressure was observed for individuals in the case group compared to controls. Individuals with type 2 diabetes had a mean age at diagnosis of diabetes 51 years with mean duration of diabetes $\sim 15$ years and similar mean blood glucose values observed between case and control groups. Average values for age at diagnosis, blood glucose and body mass index were similar between cases and controls with type 1 diabetes; both groups recorded a relatively long mean duration of diabetes ( $>25$ years).

A total of 2,765 SNPs were removed following bioinformatic evaluation with 11,152 common (minor allele frequency (MAF) >1\%) SNPs successfully analysed (Fig. 1).
Six hundred and twenty-six SNPs were observed with significance set to the five percent level (558 expected by chance alone). About 242 SNPs were observed with $P_{\text {adjusted }}$ $<0.05$ following permutation procedures to generate empirical $P$ values. SNPs were ranked for association with diabetic nephropathy according to significance values and the top ranked SNPs (Table 2) were selected for further analysis.

The top ranked SNPs were subsequently genotyped, using MassARRAY iPLEX ${ }^{\mathrm{TM}}$ GOLD (Sequenom, San Diego, CA, USA) and TaqMan (Applied Biosystems, Foster City, CA, USA) technologies, in 1,467 individuals with type 1 diabetes to investigate association with diabetic nephropathy (Table 2). There was limited support for the association between these SNPs and nephropathy in individuals with type 1 diabetes, however five SNPs revealed $P<0.05$. Two of these SNPs (rs2076484, rs9380215, $P=0.03)$ were located in $6 \mathrm{p} 21.3$.

\section{Discussion}

We have investigated 11,152 SNPs for association with diabetic nephropathy in individuals with type 2 diabetes recruited in Northern Ireland. Genotyping utilised a commercial panel that has demonstrated highly accurate and reproducible results, employing strict quality control parameters. Focusing

Table 1 Clinical characteristics of genotyped populations

\begin{tabular}{|c|c|c|c|c|}
\hline & $\begin{array}{l}\text { T2DN cases } \\
n=203\end{array}$ & $\begin{array}{l}\text { T2DN controls } \\
n=246\end{array}$ & $\begin{array}{l}\text { T1DN cases } \\
n=718\end{array}$ & $\begin{array}{l}\text { T1DN controls } \\
n=749\end{array}$ \\
\hline Age at diagnosis of diabetes (years) & $51.6 \pm 11.9$ & $51.2 \pm 9.7$ & $14.8 \pm 7.7$ & $15.4 \pm 8.0$ \\
\hline Duration of diabetes (years) & $15.2 \pm 8.4$ & $14.6 \pm 6.4$ & $34.5 \pm 9.6$ & $29.6 \pm 9.1$ \\
\hline a,b $\mathrm{HbA1c}(\%)$ & $10.2 \pm 1.7$ & $10.0 \pm 1.4$ & $8.8 \pm 1.8$ & $8.6 \pm 1.5$ \\
\hline BMI (kg/m2) & $31.5 \pm 6.6$ & $30.7 \pm 6.3$ & $26.2 \pm 5.0$ & $26.1 \pm 4.2$ \\
\hline${ }^{\mathrm{a}}$ Systolic BP (mmHg) & $147 \pm 20$ & $140 \pm 18$ & $145 \pm 21$ & $125 \pm 15$ \\
\hline${ }^{\mathrm{a}}$ Diastolic BP (mmHg) & $76 \pm 11$ & $75 \pm 10$ & $82 \pm 11$ & $75 \pm 8$ \\
\hline
\end{tabular}

Data are mean \pm SD

${ }^{a}$ values are based on the average of the most recent three measurements for each individual

b DCCT (Diabetes Control and Complications Trial) aligned assay for hemoglobin A1c

Fig. $1 \log P$ values for each SNP genotyped per chromosome

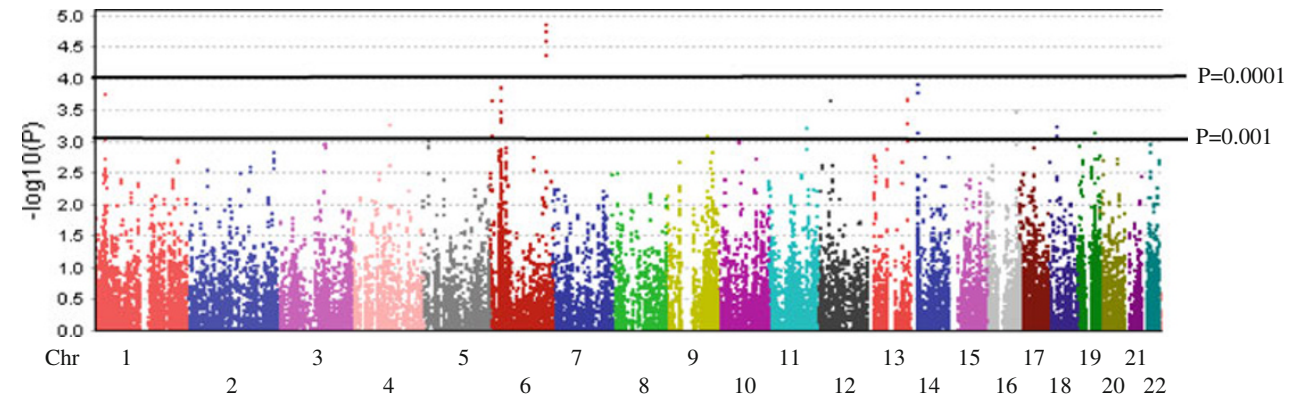




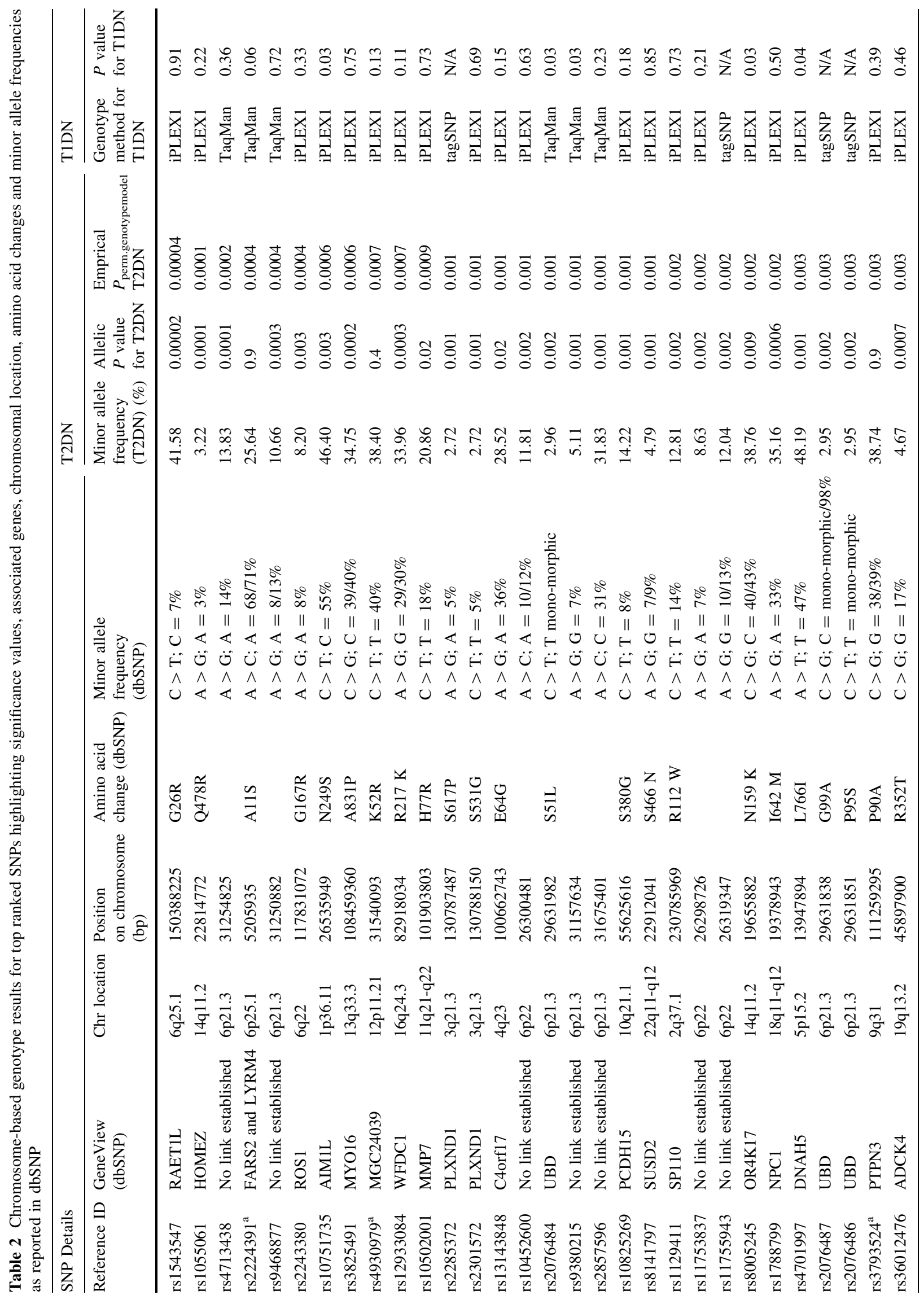




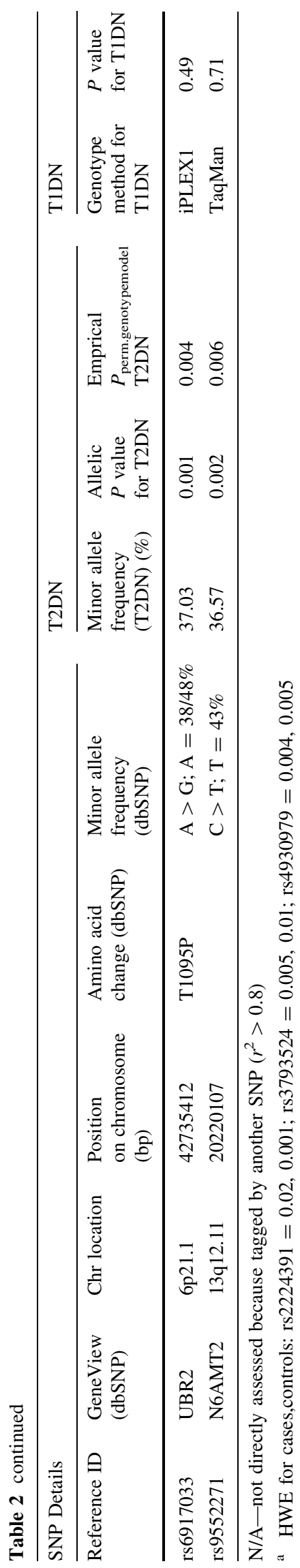

on nsSNPs is an attractive approach since less common variants that may influence the risk of disease are directly genotyped. None of the available genome-wide panels that incorporate less than a million markers are optimised to capture nsSNPs. For example, the Affymetrix $500 \mathrm{~K}$ chip covers only $65 \%$ of the common nsSNPs ( $\geq 5 \%$ MAF) and $24 \%$ of the rarer variants $(<5 \% \mathrm{MAF})$ present on the HNS12 panel with an $r^{2}>0.8$ in the CEU population (Evans et al. 2008). The Ensembl genome browser contains 97,571 nsSNPs (www.ensembl.org, accessed 08/06/09) with a recent report describing 133,505 coding SNPs in the human genome (Reumers et al. 2008), although it should be noted that many of these are not validated.

The top ranked SNPs, with respect to the adjusted $\mathrm{P}$ value, were located in several biological and positional candidate genes for diabetic nephropathy. rs 1543547 demonstrated the most evidence for association (adjusted $P_{\text {genotype }}=4 \times$ $10^{-5}, P_{\text {allelic }}=2 \times 10^{-5}$ ) based on comparisons of genotype and allele frequencies between cases and controls. This SNP is located at 6q25.1 in the retinoic acid early transcript $1 \mathrm{~L}$ gene. RAETIL spans approximately $5 \mathrm{~kb}$ and is a member of the RAET1 family of MHC class I-related genes located within the $180 \mathrm{~kb}$ cluster on chromosome 6q24.2$6 \mathrm{q} 25.3$. Fine mapping would be complex as genes within this region are very similar; for example, RAETIL reveals approximately $97 \%$ identity with $U L B P 2$ (Radosavljevic et al. 2002). Information is sparse regarding the precise function of this gene, however RAETI genes encode glycoproteins that are anchored to the membrane via glycosylphosphatidylinositol linkage (Radosavljevic et al. 2002).

Within the top ranked group, two nsSNPs were clustered in the PLXND1 gene (rs2285372, rs2301572) at 3q21.3. $P L X N D 1$ is expressed in human vascular endothelial cells and is important for cardiovascular development (Gitler et al. 2004). Eight of the top ranked 32 SNPs were located at 6p21.3 with three nsSNPs clustered within the ubiquitin D $(U B D)$ gene at 6p21.3 (rs2076487, rs2076486, rs2076484).

Several differences were observed when comparing our genotype data to the higher-throughput information recorded in dbSNP (www.ncbi.nlm.nih.gov/snp). Two SNPs recorded in dbSNP as monomorphic (rs2076486, rs2076848) were observed with minor allele frequencies of $3 \%$. SNP rs 1543547 was observed with $42 \%$ minor allele frequency in our population compared to the $7 \%$ reported for the HapMap CEU population. However, our results for rs 1543547 are similar to those reported by Perlegen (ss24453248) where the T allele was observed at $46 \%$ in 24 samples from the Coriell Cell Repository that were primarily of European descent. Linkage disequilibrium $\left(r^{2}>0.8\right)$ was observed between: rs2076486, rs2076484, and rs2076487; rs2301572 and rs2285372; rs11755943 and rs 10452600 . These relationships were exploited by only selecting tag SNPs for further genotyping, 
Ideally our results would be replicated in a larger, phenotypically similar group of individuals from the same geographical region; such a collection is not available within the British Isles. As there are shared pathophysiological mechanisms contributing to the development of diabetic kidney disease (for example, mesangial expansion and glomerular basement membrane thickening) in those with type 1 and type 2 diabetes (Caramori and Mauer 2003; Tsilibary 2003; Fioretto and Mauer 2007), we also genotyped the top ranked variants to evaluate association with type 1 diabetes. This larger case-control collection $(n=1,467)$ recruited individuals from Ireland in conjunction with a UK multicentre collection (Genetics Of Kidneys in Diabetes UK) that was specifically designed to investigate genetic risk factors for diabetic nephropathy (McKnight et al. 2009b). A tag SNP approach was taken where strong linkage disequilibrium was observed, resulting in 28 SNPs actually genotyped in the follow-up study. Although a strong association was not observed with nephropathy in type 1 diabetes, two SNPs in 6p21.3 (rs2076484, rs9380215, $P=0.03$ ) warrant further investigation.

It is possible that the results from individuals with type 2 diabetes in Table 2 represent type 1 errors, or that genetic variants associated with nephropathy in type 1 diabetes may differ from those involved with nephropathy in type 2 diabetes. Robust quality control procedures were employed and several SNPs in close proximity to each other were associated with diabetic nephropathy in biologically plausible genes. Replication and fine mapping in large populations of phenotypically similar individuals are required to investigate further the top ranked variants.

Non-synonymous SNPs contribute to inter-individual differences, the variation within biological networks, genetic susceptibility to disease, and prediction of drug responsiveness (Karchin et al. 2005). We have completed a targeted genome-wide scan and revealed several novel SNPs in genes that are worthy of further investigation; validation studies are essential to confirm the importance of these findings.

\section{Methods}

Ethical approval was obtained from the appropriate Research Ethics Committee and all participants provided written, informed consent prior to enrolment. All individuals recruited for this study were White, with parents and grandparents born in the British Isles.

Individuals with type 2 diabetes

About 449 individuals (203 cases, 246 controls) were diagnosed with type 2 diabetes and genotyped in the original panel. Type 2 diabetes was defined as current or previous use of hypoglycaemic agents or diet without recourse to insulin in the first year of diabetes diagnosis; MODY patients were excluded. Cases with diabetic nephropathy were defined as such if they had persistent proteinuria with $(>0.3 \mathrm{~g}$ protein $/ 24 \mathrm{~h}$ or raised albumincreatinine ratio (ACR) $>30 \mathrm{mg} / \mathrm{mmol}$ on at least two occasions), or previously had levels of proteinuria consistent with this case definition prior to commencement of renin-angiotensin system blocking drugs. Cases with diabetic nephropathy may have had chronic renal failure (eGFR $<60 \mathrm{ml} / \mathrm{min} / 1.73 \mathrm{~m}^{2}$ ) or end stage renal disease (managed by dialysis or renal transplant). Importantly, patient notes and previous consultant medical opinions were scrutinised to exclude potential cases with non-diabetic nephropathy. The control group consisted of individuals with type 2 diabetes of at least 10 years duration and who had no evidence of proteinuria or microalbuminuria on repeated testing. Individuals receiving ACE inhibitors and/or angiotensin receptor blockers were excluded from the control group; this helps to avoid misclassification of the control phenotype since anti-hypertensive treatment may lower urinary albumin excretion.

Individuals with type 1 diabetes

This collection includes individuals recruited as part of the Warren 3 and GoKinD UK resources (McKnight et al., 2009b). All individuals were diagnosed with type 1 diabetes before 31 years of age. Individuals with nephropathy had diabetes for at least 10 years before the onset of persistent proteinuria $(>0.5 \mathrm{~g}$ protein $/ 24 \mathrm{~h})$, hypertension (blood pressure $>135 / 85 \mathrm{mg} \mathrm{Hg}$ and/or treatment with antihypertensive medication) and diabetic retinopathy. Individuals recruited as controls had duration of diabetes of at least 15 years, were not taking antihypertensive medication and did not demonstrate any evidence of renal disease. The presence of microalbuminuria (20-200 mcg/min) or evidence of non-diabetic renal disease were exclusion criteria for this collection.

Genotyping and data analysis

The commercially available Human NS12 BeadChip was processed using the Infinium ${ }^{\circledR}$ I Whole-Genome Genotyping Assay (Illumina Inc, San Diego, CA, USA, www.illumina.com). This HNS12 panel contains 13,917 unique SNPs, developed from the targeted investigation of high-value, gene-centric variants, and comprises more than $11,600 \mathrm{nsSNPs}$ distributed across the genome together with a dense set of 870 tag SNPs across the MHC region. Genotyping of the HNS12 panel was conducted at deCODE Genetics (Reykjavik, Iceland); further details 
regarding the assays are available from the authors. SNPs with individual GenCall scores $>0.25$, call rates of at least 90\% for both cases and controls, genotype distributions in accordance with Hardy-Weinberg equilibrium, and minor allele frequencies $>1 \%$ were compared between case and control groups. Comparison of cases and controls was conducted using PLINK version 1.04 (Purcell et al. 2007; McKnight et al. 2009b). Permutation procedures provide a framework to adjust for multiple comparisons and empirical $\mathrm{P}$ values were generated using the permutation procedure (genotype model) implemented in PLINK. A quantile-quantile plot of observed chi-squared test statistics against expected order statistics was used to assess possible inflation of the chi-squared test statistics (Clayton et al. 2005). The current cytogenetic and sequence position on each chromosome location was obtained from dbSNP build 129, GenBank genome build 36.3, along with amino acid changes in associated genes.

Linkage disequilibrium was investigated for the top ranked SNPs and tag SNPs identified $\left(r^{2}>0.8\right)$ using Haploview (Barrett et al. 2005; de Bakker et al. 2005). Genotyping was performed for the top ranked variants in the type 1 diabetic collection using iPLEX (Sequenom Compact, San Diego, CA, USA) and TaqMan (7900HT, Applied Biosystems, Warrington, UK) technologies. Cases and controls were randomly arranged in 384-well plates with uniquely positioned negative controls, duplicate samples and four father-mother-proband trios providing experimental quality control. As above, genotypic and allelic data were compared for cases and controls using PLINK.

Acknowledgments We are greatly indebted to all participants as well as the medical staff who collected resources. In particular, we acknowledge the nurses who recruited patients: Valerie Millar, Rebecca Kikkert, Julie Lewis, Pamela Strong, Michelle McKinley, and Michael Matthews; the consultant nephrologists in Northern Ireland, and Drs Henry, Archbold, Loughrey, McConnell and Rooney in the Diabetes clinics for assistance in identifying patients. This work was financially supported by the Northern Ireland Kidney Research Fund and The Research and Development Office of Northern Ireland. The authors wish to thank Nanna Viðarsdóttir and Joseph Prosser for facilitating HNS12 genotyping at deCODE Genetics (Reykjavik, Iceland). The Warren 3/UK GoKinD Study Group was jointly funded by Diabetes UK and the Juvenile Diabetes Research Foundation. The Warren 3/UK GoKinD Study Group includes, Belfast: A. P. Maxwell, A. J. McKnight, D. A. Savage; Edinburgh: J. Walker; London: S. Thomas, G. C. Viberti; Manchester: A. J. M. Boulton; Newcastle: S. Marshall; Plymouth: A. G. Demaine, B. A. Millward; Swansea: S. C. Bain.

Funding Northern Ireland Kidney Research Fund and The Research and Development Office of Northern Ireland.

\section{References}

Ansell D, Feehally J, Fogarty D et al (2008) UK Renal Registry Report 2008. UK Renal Registry, Bristol, UK
Barrett JC, Fry B, Maller J, Daly MJ et al (2005) Haploview: analysis and visualization of LD and haplotype maps. Bioinformatics 21:263-265

Caramori ML, Mauer M (2003) Diabetes and nephropathy. Curr Opin Nephrol Hypertens 12:273-282

Clayton DG, Walker NM, Smyth DJ et al (2005) Population structure, differential bias and genomic control in a large-scale, casecontrol association study. Nat Genet 37:1243-1246

de Bakker PI, Yelensky R, Pe'er I et al (2005) Efficiency and power in genetic association studies. Nat Genet 37:1217-1223

Evans DM, Barrett JC, Cardon LR (2008) To what extent to scans of non-synonymous SNPs complement denser genome-wide association studies? Eur J Hum Genet 16(6):718-723

Fioretto P, Mauer M (2007) Histopathology of diabetic nephropathy. Semin Nephrol 27(2):195-207

Gitler AD, Lu MM, Epstein JA (2004) PlexinD1 and semaphorin signaling are required in endothelial cells for cardiovascular development. Dev Cell 7:107-116

Hampe J, Franke A, Rosenstiel P et al (2007) A genome-wide association scan of nonsynonymous SNPs identifies a susceptibility variant for Crohn disease in ATG16L1. Nat Genet 39: 207-211

Karchin R, Diekhans M, Kelly L et al (2005) LS-SNP: large scale annotation of coding non-synonymous SNPs based on multiple information sources. Bioinformatics 21(12):2814-2820

McKnight AJ, O'Donoghue D, Maxwell AP (2009a) Annotated chromosome maps for renal disease. Hum Mutat 30(3):314-320

McKnight AJ, Woodman AM, Parkkonen M et al (2009b) Investigation of DNA polymorphisms in SMAD genes for genetic predisposition to diabetic nephropathy in patients with type 1 diabetes mellitus. Diabetologia 52(5):844-849

Purcell S, Neale B, Todd-Brown K et al (2007) PLINK: a tool set for whole-genome association and population-based linkage analyses. Am J Hum Genet 81(3):559-575

Radosavljevic M, Cuillerier B, Wilson MJ et al (2002) A cluster of ten novel MHC class I related genes on human chromosome 6q24.2-q25.3. Genomics 79:114-123

Reumers, Conde L, Medina I, et al. (2008) Joint annotation of coding and non-coding single nucleotide polymorphisms and mutations in the SNPeffect and PupaSuite databases. Nucleic Acids Research 36(database issue): D825-829

Savage DA, Bain SC, McKnight AJ et al (2007) Gene discovery in diabetic nephropathy. Curr Diab Rep 7(2):139-145

Savage DA, Patterson CC, Deloukas P et al (2008) Genetic association analyses of non-synonymous single nucleotide polymorphisms in diabetic nephropathy. Diabetologia 51(11): 1998-2002

Smyth DJ, Cooper JD, Bailey R et al (2006) A genome-wide association study of nonsynonymous SNPs identifies a type 1 diabetes locus in the interferon-induced helicase (IFIH1) region. Nat Genet 38:617-619

Stitziel NO, Binkowski TA, Tseng YY, et al. (2004) TopoSNP: a topographic database of non-synonymous single nucleotide polymorphisms with and without known disease association. Nucleic Acids Res 32(Database issue): D520-522

Tsilibary EC (2003) Microvascular basement membranes in diabetes mellitus. J Pathol 200(4):246-537

USRDS, U.S. Renal Data System (2008) Annual data report: atlas of chronic kidney disease and end-stage renal disease in the United States, National Institutes of Health, National Institute of Diabetes and Digestive and Kidney Diseases, Bethesda, MD

WTCCC (2007) The wellcome trust case control consortium. Association scan of 14, 500 nonsynonymous SNPs in four diseases identifies autoimmunity variants. Nat Genet 39:1329-1337 